\title{
Sozial Benachteiligte sind auch kränker
}

\author{
Daniel Lüthi \\ Freier Journalist und Fotograf, Medientrainer, Bern
}

\begin{abstract}
"Arme leben kürzer als Reiche und sind häufiger krank»: Dies schreibt die WHO bereits 2004 in einer umfassenden Untersuchung mit dem Titel Soziale Determinanten von Gesundheit. Mit anderen Worten: Soziale Ungleichheiten sind ein Hauptgrund für gesundheitliche Ungleichheit. Aktuelle Studien und Projekte in der Schweiz bestätigen dies.
\end{abstract}

Eben sind die jüngsten Ergebnisse der Schweizerischen Gesundheitsbefragung herausgekommen, die das Bundesamt für Statistik (BfS) seit 1992 durchführt. Themen sind unter anderem körperliche Aktivitäten, Tabak- und Alkoholkonsum oder Ernährung - und damit Übergewicht und Fettleibigkeit. Ein Beispiel, wo als beeinflussende Faktoren nicht bloss genetische Veranlagungen eine wichtige Rolle spielen, sondern auch soziale Elemente: Bildungsstand, Einkommen, soziale Integration, Lebens- und Arbeitsbedingungen, Gesundheitsverhalten und kulturelle Gewohnheiten.

\section{Beispiel Übergewicht}

In der Auswertung seiner Gesundheitsbefragung nennt das BfS ein gewichtiges Beispiel: "Bei der Adipositas besteht ein erheblicher sozialer Gradient. Das Adipositasrisiko ist bei Personen ohne nachobligatorische Ausbildung deutlich grösser als bei Personen mit einem Hochschulabschluss.» Damit steigt bei Menschen mit einem geringeren Bildungsniveau auch das Risiko für Herz-Kreislauf-Erkrankungen, Diabetes, gewisse Krebsleiden (besonders Brust- und Dickdarmkrebs) sowie Erkrankungen des Bewegungsapparates wie zum Beispiel Arthrose. BfS-Fachmann Marco Storni erklärt dazu: «Soziale Unterschiede lassen sich in vielen Bereichen feststellen, sowohl im Gesundheitsverhalten als auch im Gesundheitszustand oder auch bei Arztbesuchen.» Ausschlaggebend seien insbesondere Aspekte der Prävention: «Sozial besser gestellte Personen zeigen in vielen Belangen - wie z.B. Rauchen, Ernährung und Bewegung - öfters ein gesundes Verhalten als sozial weniger gut gestellte Personen.»

\section{Chancengleichheit und Gesundheit}

Auch das Bundesamt für Gesundheit (BAG) hat zum Thema gerade neue Zahlen und Fakten veröffentlicht, dies unter dem Titel «Chancengleichheit und Gesundheit». Zwei beeindruckende Erkenntnisse daraus: «Die Lebenserwartung unterscheidet sich nach dem Wohnort. Je tiefer der sozioökonomische Status in einer Gemeinde bzw. in einem Quartier, desto tiefer die Lebenserwartung.» Und: "Stark Rauchende kommen überdurchschnittlich häufig aus Schichten mit einem tieferen Bildungsniveau. Das Risiko, an Lungenkrebs zu erkranken, ist für Menschen mit tieferem Einkommen fast sechs Mal höher als für solche mit höherem Einkommen.»

Die Untersuchung des BAG zeigt übrigens auch, dass ein guter Bildungsstand und ein ausreichendes Einkommen nicht nur die physische, sondern auch die psychische Gesundheit unterstützen.

\section{Und jetzt?}

Was ist zu tun, um die Chancengleichheit zu erhöhen? Die Bemühungen hätten sich bisher vor allem auf den Migrationsbereich konzentriert, schreibt das BAG dazu. Stichwort: interkulturelle Dolmetscherinnen und Dolmetscher. Gibt es neue Tendenzen? Karin Gasser, Co-Leiterin der Sektion Gesundheitliche Chancengleichheit im BAG, bleibt vage: «Es wird darauf hingewirkt, dass die nationalen Public-Health-Strategien so umgesetzt werden, dass sie benachteiligte Bevölkerungsgruppen berücksichtigen. Wir wollen auch klären, wie wir diese Gruppen am besten erreichen - mit 
welchen Botschaften und via welche Kanäle. 2019 wird eine Publikation veröffentlicht, die die Erfolgsfaktoren chancengleicher Prävention und Gesundheit aufzeigt.» Neu sei auch die Gruppe der Armutsbetroffenen - darunter die Sozialhilfeempfänger - in den Fokus gerückt. «Das Problem ist, dass in der Schweiz in dieser Thematik repräsentative Studien fehlen.»

\section{Und die Ärztinnen und Ärzte?}

Konkret setze das BAG selber kaum Projekte um, erklärt dessen Vertreterin: «Für diese Frage muss an die Leistungserbringer verwiesen werden.»

Fragen wir die Ärztin, Wissenschaftlerin und PublicHealth-Spezialistin Julia Dratva. Bei der ZHAW leitet sie die Forschungsstelle Gesundheitswissenschaften, und sie ist Präsidentin der Schweizerischen Gesellschaft der Fachärztinnen und -ärzte für Prävention und Gesundheitswesen, SGPG. Grundsätzlich gelte es, sagt Dratva, «die Balance zu halten zwischen den medizinisch-technischen Möglichkeiten und dem Bedarf von Menschen, mit ihren Ärztinnen und Ärzten zu kommunizieren». Personalisierte Medizin also? «Personalisierte Medizin heisst leider nicht, dass die Medizin persönlicher würde, sondern nur spezifischer. Die Tendenz, das eine höher zu werten - auch tariflich und das andere weniger, ist eine Sackgasse.» Immer wieder rückt die Prävention in den Fokus. Dazu erklärt Julia Dratva: «Politisch wird gerne die Verhal- tensprävention, die die Verantwortung dem Einzelnen zuschiebt, gepredigt. Als Ärzte und Ärztinnen müssen wir individualmedizinisch Verantwortung übernehmen, aber auch bevölkerungsmedizinisch. Ärzte und Ärztinnen sollten sich als Advokaten der Bevölkerung verstehen und sich auch politisch für gesunde Lebensbedingungen aller und insbesondere vulnerabler Gruppen einsetzen.»

Weil es eben nicht nur um Gesundheit geht, sondern auch um Chancengleichheit.

\section{forumsante.ch}

Am Dienstag, 15. Januar, findet im Hotel Bellevue in Bern das forumsante.ch bereits zum 20. Mal statt. Die Tagung lädt zu einer vielschichtigen Diskussion über ein aktuelles Thema: "Sozioökonomische Determinanten - sag mir, wer du bist, und ich sage dir wie lange du lebst.»

Renommierte nationale und internationale Referenten setzen sich aus medizinischer, wirtschaftlicher, politischer und ethischer Perspektive mit der Leitfrage auseinander und beleuchten auch die Rolle der Gesundheitsprofis.

Diskutiert werden soll aber nicht nur die Gegenwart: forumsante. ch schaut immer einen Schritt voraus und bildet eine Plattform, um über konstruktive Ideen und pointierte Aussagen nachzudenken und gemeinsam über die Zukunft zu diskutieren. Website mit vollständigem Kongressprogramm und Anmeldung: www.forumsante.ch

Korrespondenz: forumsante[at]furrerhugi.ch 